\title{
A Fully Distributed Coordination Scheme Based on Orthogonal Requests and Responses
}

\author{
Seungkwon Cho***, Sungcheol Chang*, Youngnam Han** \\ * ETRI(Electronics and Telecommunications Research Institute), Korea \\ ** Department of Electrical Engineering, KAIST(Korea Advanced Institute of Science and Technology), Korea \\ skcho@etri.re.kr
}

\begin{abstract}
Device-to-device (D2D) communication is a communication technology that enables one device to transmit data directly to the other device in proximity instead of conveying data via infrastructure. Without the coordination of infrastructure, multiple access for D2D communication is performed in a distributed manner. Even though contentionbased distributed multiple access scheme is widely adopted in the existing D2D systems, the excessive contention overhead with a large number of devices makes contention-based scheme lose scalability. In this paper, we present the rationale behind the design of a new fully distributed multiple access scheme based on contention-free resource request and response message exchange, which is proposed for IEEE 802.15.8. Through the results of simulations with full buffer and VoIP traffic model, we show that the presented scheme achieves scalability with the help of orthogonal requests and responses
\end{abstract}

Keywords - D2D, Multiple access, Distributed Scheduling, IEEE 802.15.8, PAC.

\section{INTRODUCTION}

Recently, a new standard for D2D communication among devices in proximity is being developed by IEEE 802.15.8 Task Group. The purpose of this Task Group is to provide infrastructure-less peer aware communication (PAC) with fully distributed coordination. Even though there are a lot of achievable features of PAC, scalability is the key feature which distinguishes it from other existing D2D systems. PAC considers discovery of up to 1000 devices and data communication among a subset of those devices [1].

Scalability in a wireless communication system with contention-based distributed coordination is less likely to be achieved without increase in channel access latency. It is because collisions in channel access increases rapidly as the number of contending device increases and thus longer backoff time results in an inevitable increase in channel access latency. Contention-free distributed coordination is an alternative and suitable scheme for avoiding excessive contention overhead inherent in contention-based distributed coordination. Collision-less channel access attempt in distributed coordination means that resource used to try channel access is assigned to each device in a predetermined manner. Thus, without central coordination, contention-free distributed coordination implicitly requires synchronization among devices.

FlashLinQ [2] is one example of contention-free fully distributed coordination. The term "fully distributed coordination" is used in this paper to refer to a coordination scheme of which scheduling decision is made individually by each device with same scheduling algorithm. Contention-free analogue signaling of FlashLinQ is enabled by a pair of single tones mapped to each D2D link in non-overlapping manner after link setup. FlashLinQ device can transmit data after successful scheduling based on SIR(Signal to Interference Ratio) measurement by exchanging analogue tones. Even though SIR measurement-based scheduling with orthogonal tones has beneficial effect on scalability, it causes cascade yielding problem [3] which refers to unnecessary yield of data transmission opportunity. Moreover, OFDMA-based analogue signaling of FlashLinQ is hard to be adopted for a distributed network because frequency synchronization of FlashLinQ is derived from cellular base station.

A new fully distributed coordination scheme [4] is proposed for IEEE 802.15.8. In the proposed scheme, orthogonal time-domain requests and responses is utilized in order to avoid significant performance deterioration in a dense device environment. In this paper, rationale behind the design of the proposed scheme and performance result focused on scalability is presented.

The rest of this paper is structured as follows. Section II describes details of a fully distributed coordination scheme presented in this paper. We provide simulation results and discussions in Section III. Section IV concludes this paper.

\section{The CONTENTION-FREE Fully DisTRIBUTED MULTIPLE ACCESS SCHEME}

The presented distributed coordination scheme in this paper is part of proposal submitted to IEEE 802.15.8. In the submitted proposal [4], not only multiple access scheme but also other fundamental functionalities of D2D communication such as distributed synchronization, discovery, and peering are also proposed. However, the scope of this paper is limited to the proposed multiple access scheme to provide the rationale behind it and its performance with respect to scalability. To concentrate on multiple access scheme, description of other part of the proposal is briefly described. 


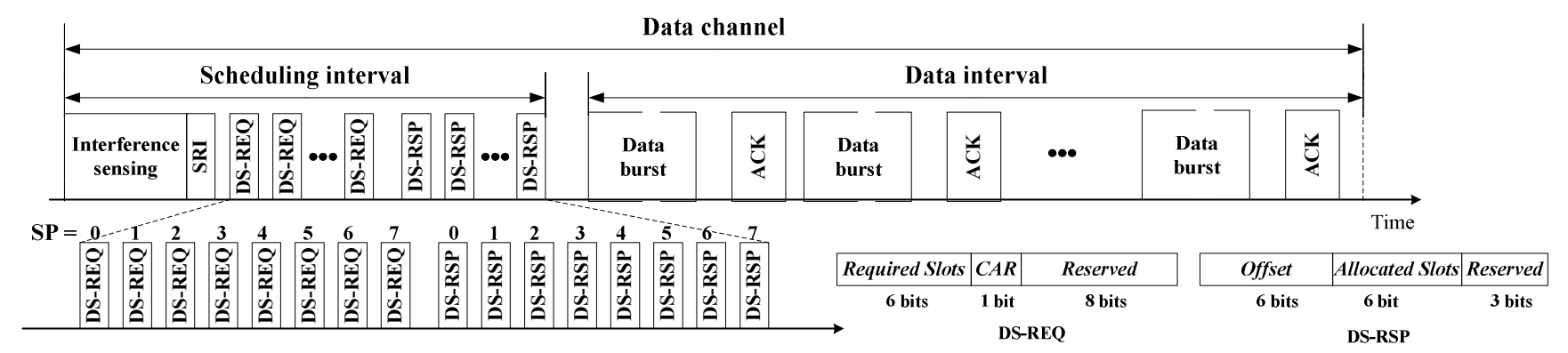

Figure 1. Data channel structure

\section{A. An Overview of Device Operation}

The Radio resource is divided in time by its usage such as synchronization region, discovery region, peering region, and data region and each region, which is used for distributed synchronization, device/service discovery, peering, and data communication, respectively. After powered up, a device tries to synchronize in a distributed manner. Once synchronization is achieved, each device initiates and continues discovery process to identity devices and/or services in proximity. The peering process is a link setup process, between devices discovered during the discovery process, enabling the peered devices to be ready to exchange data. After peering, a locally unique peering ID (PID) is shared between the peered devices. Only peered devices can try to access the data region according to the coordination scheme presented in this paper.

\section{B. Frame Structure}

A superframe is comprised of one Type 0 frame and 9 Type 1 frames. A Type 0 frame has all the regions such as synchronization region, discovery region, peering region, and data region. A Type 1 frame has only data regions. Data region is comprised of a series of multiple data channels. There are 13 and 16 data channels in Type 0 frame and Type 1 frame, respectively.

A data channel is a fundamental radio resource where ctual data transmissions take place and it consists of scheduling interval and data interval as is illustrated in Figure 1. In a scheduling interval, time interval for interference sensing is followed by resource for SRI (Scheduling Request Indicator), DS-REQs (Distribute Scheduling - Requests) and DS-RSPs (Distribute Scheduling - Responses). Interference sensing is for coexistence in unlicensed band. An originator senses the air medium to check whether there are interferences from heterogeneous device. The originator refers to a device trying to access data interval. If the air medium is determined to have no interference, the originator transmits SRI before transmitting DS-REQ. Transmission of SRI signal is related to consecutive allocation which will be explained later in this section. DS-REQ is transmitted by an originator and DS-RSP is transmitted by a recipient in a response to the received DSRSP. The recipient refers to the peer of the originator. Since there are 8 DS-REQ and DS-RSP pairs in a scheduling interval, a maximum of 8 transmissions can be concurrently scheduled for a data interval.
Each scheduled transmission in a data interval has resource for both data burst and ACK which is transmitted by the originator and the recipient, respectively. A dedicated resource for ACK is allocated after one exchange of DS-REQ and DSRSP to avoid excessive scheduling overhead.

\section{Resource Mapping}

The mapping from the PID in conjunction with synchronization information such as frame number and superframe number to available resource for DS-REQ and DS-RSP is referred to as resource mapping. Two hierarchical mappings are required for resource mapping; one is data channel mapping and the other is SP (Scheduling Priority) mapping. Notice that the guarantee of sharing locally orthogonal PID after peering process allows peered devices to have non-overlapping resources for DS-REQ and DS-RSP, which results in contention-free distributed coordination.

Available data channels for the given PID are assigned by data channel mapping. The available data channel index $l(0 \sim$ $15)$ for the peered devices with PID $p(0 \sim 127)$, frame number $n(0 \sim 9)$, and superframe number $s(0 \sim 15)$ is given by

$$
l=(\text { floor }(p / 8)+s \times 10+n) \text { modulo16. }
$$

Notice that, data channels corresponding to $l=0,1,2$ are not defined in Type 0 frame $(n=0)$.

In an assigned data channel, resources for DS-REQ and DS-RSP are determined by SP (Scheduling Priority) mapping based on the PID. As is shown in Figure 1, SP ranges from 0 to 7 and the rightmost resource for DS-RSP and DS-REQ corresponds to the highest SP. The assigned SP for the peered devices is given by

$$
\mathrm{SP}(p, n, s)=\sum_{k=1}^{(p+s \times 10+n) \text { modulo } 8}(-1)^{k+1} \cdot(8-k)
$$

if $(p+s \times 10+n)$ modulo $\neq 0$, and

$$
\operatorname{SP}(p, n, s)=0
$$

otherwise.

\section{Distributed Scheduling}

Required slots field of DS-REQ payload in Figure 1 is set to the required resource in the data interval in terms of OFDM slots. OFDM slot is a basic unit of data interval and one OFDM slot is 4 OFDM symbols. The required resource includes data burst, ACK, guard interval (GI) between data burst and ACK, and GI after ACK.

Since all the recipients exactly know the time when its peered originator can send DS-REQ, all the recipients can 
keep monitoring DS-REQ transmitted by its peered originator. If the recipient failed to decode DS-REQ payload, the recipient does not need to receive any other DS-REQs and waits for the next DS-REQ from its peered originator in the next mapped data channel. Otherwise, the recipient receives all the following DS-REQs with higher SP than its own and accumulates the value of Required slots field of all the received DS-REQs with higher SP than its own.

The recipient transmits DS-RSP in a response to the received DS-REQ with Offset field set to the accumulated value of Required slots field of all the received DS-REQs with higher SP than its own. Notice that resource for DS-RSP specified by SP mapping, which means DS-RSP is also transmitted in a contention-free manner. The recipient does not send DS-RSP if the Offset field exceeds data interval boundary. Thus scheduling request from device with higher SP has better change of allocation rather than those from device with lower SP.

The other field of DS-RSP is Allocated slots field. Basically, Allocated slots field of DS-RSP payload is same to the Required slots field of the DS-REQ received from the originator. Allocated slots filed is adjusted to meet data interval boundary only when (Offset + Required slots) exceeds the boundary.

After transmitting DS-REQ, the originator tries to receive DS-RSP from its recipient. If the originator fails to receive DS-RSP from its recipient, it stops scheduling process and cannot transmit anything in the data interval. Otherwise, the originator has to receive the following DS-RSPs from other devices with higher SP than its own. The final step of scheduling process for the originator is to check to see whether its allocated resource ranging from Offset to (Offset + Allocated slots) overlaps with resources allocated to other devices with higher SP than its own. Only when there is no overlap, the originator can access data interval and transmit its data burst at the scheduled Offset. The recipient transmits ACK at (Offset + Allocated slots - ACK duration - GI between ACK and the next data burst) to signify

TABLE 1. SIMULATION PARAMETERS

\begin{tabular}{|c|l|}
\hline Parameter & \multicolumn{1}{|c|}{ Value } \\
\hline Frame length & $20 \mathrm{msec}$ \\
\hline Data channel length & $1.232 \mathrm{msec}$ \\
\hline Length of scheduling interval & $0.256 \mathrm{msec}$ \\
\hline Length of data interval & $0.96 \mathrm{msec}$ \\
\hline $\begin{array}{c}\text { GI between scheduling interval and data } \\
\text { interval }\end{array}$ & $16 \mathrm{usec}$ \\
\hline $\begin{array}{c}\text { GI between data burst and ACK } \\
\text { GI between ACK and the next data burst } \\
\text { OFDM symbol length }\end{array}$ & $16 \mathrm{usec}$ \\
\hline $\begin{array}{c}\text { Number of data subcarriers per OFDM } \\
\text { symbol }\end{array}$ & $4 \mathrm{usec}$ \\
\hline $\begin{array}{c}\text { Modulation and coding scheme(MCS) for } \\
\text { data }\end{array}$ & $64 \mathrm{subcarriers}$ \\
\hline $\begin{array}{c}\text { Number of OFDM symbols used for } \\
\text { preamble in data burst }\end{array}$ & 2 OFDM symbols \\
\hline $\begin{array}{c}\text { MCS for DS-REQ/DS-RSP/ACK } \\
\text { Length of DS-REQ/DS-RSP/ACK }\end{array}$ & BPSK, 1/2 \\
\hline
\end{tabular}

acknowledgement. On the contrary, if any overlaps are found, the originator cannot access the data interval. The rationale behind this post-scheduling process is to protect data burst transmission with higher SP from other transmission by hidden-node devices with lower priority.

\section{E. Consecutive Allocation}

Let us define normal allocation as an allocation mechanism which facilitates resource allocation only in a data channel according to the aforementioned distributed scheduling. Only with normal allocation, since available data channels for a pair of peered device with specific PID do not appear back-to-back by the resource mapping, any devices mapped to $l$-th data channel cannot even try to access the following $(l+1)$-th data channel. This limitation can cause resource underutilization when all the devices mapped to $(l+1)$-th data channel have no data to transmit but the devices mapped to $l$-th data channel still have something to transmit.

Consecutive allocation is the other allocation mechanism enabling a device to have an opportunity of another normal allocation in the following data channel. To try consecutive allocation in $(l+1)$-th data channel, the originator sets CAR (Consecutive allocation request) bit to 1 when it sends DSREQ to the recipient in $l$-th data channel. From the viewpoint of the recipient, if CAR bit of the received DS-REQ is set to 1, it has to participate in consecutive allocation in the next data channel. If the originator fails to receive DS-RSP from the recipient in $l$-th data channel, it stops all the consecutive allocation process because the recipient has a high probability of failure in successful CAR notification.

In $(l+1)$-th data channel, after interference sensing, both the originator and the recipient try to detect SRI signal. They have to immediately stop consecutive allocation process when SRI signal is detected. Detection of SRI means that there is at least a pair of peered devices eligible for normal allocation in $(l+1)$ th data channel according to the data channel mapping. If SRI signal is not detected, they proceed with consecutive allocation of which procedure is same to that of normal allocation.

\section{III.SIMULATION RESULTS AND DISCUSSIONS}

We investigated the performance of the presented coordination scheme through computer simulation. Focused on scalability, performance evaluation is carried out in terms of mean throughput per device and mean packet latency. The mean throughput per device is expressed in bits per second (bps) and defined as the total amount of data successfully received by a recipient normalized by total simulation time. The mean packet latency refers to the average time required for user data between the arrival at transmission queue of device and the successful transmission. In this paper, Most of the simulation scenarios and parameters including channel models comply with the evaluation methodology [1] specified by IEEE 802.15 .8 . Other simulation parameters specific to this paper are listed in Table 1. Notice that this paper concentrates on investigating scalability with respect to the presented coordination scheme assuming TDMA/OFDM operation. 
Such concentration on coordination scheme leads us to assume that there are no heterogeneous devices and no errors in the reception of SRI signal. Furthermore, we also assume that all the OFDM symbols except those used for preamble in data burst are utilized for user data delivery.

Figure 2 shows mean throughput per device for the given number of devices in $500 \times 500$ square meter area under full buffer traffic model. The left-upper point of line in Figure 2 corresponds to the case when the number of devices is 2 and the mean throughput per device is $43.2785 \mathrm{Mbps}$ which corresponds to the maximum achievable throughput. At first glance the throughput per device rapidly decreases as the number of devices increases from 2 to 200. The reason behind the rapid decrease in left part Figure 2 can be revealed by more careful observation with respect to device density in terms of the number of devices per unit square meter. The device density increases by 50 times when the number of devices increases from 2 to 100 . However, device density increases by 1.12 times when the number of devices increases from 800 to 900 , respectively. A swift increase of device density means more and more pairs of devices participate in distributed scheduling, which results in rapid decrease in mean throughput per device. Thus, the mean throughput per device decreases steeply and then becomes gentle as the number of devices increases in Figure 2. Notice that, even with 1024 devices, 2.09Mbps of mean throughput is still supported with the presented coordination scheme.

Figure 3 is the investigated mean packet delay of VoIP traffic with the 2-state Markov model specified in [5]. Assuming $50 \%$ voice activity factor, ON and OFF time is exponentially distributed with mean $1.25 \mathrm{sec}$. A VoIP packet is generated every $20 \mathrm{msec}$ by AMR vocoder in the ON state and the VoIP packet size is assumed to be 42 bytes after header compression. 14 bytes of comport noise packet is also generated every $160 \mathrm{msec}$ in the OFF state. It is interesting that that packet latency remains almost constant at $23 \mathrm{msec}$ regardless of the investigated number of devices. As we mentioned earlier, the presented coordination scheme can schedule a maximum of 8 originators at one time if the data interval is enough sufficient for the sum of required resources. Therefore we can notice that the small VoIP packets from multiple devices are scheduled at one distributed scheduling without introducing too much scheduling overhead. This lowoverhead scheduling has beneficial effect on scalability with respect to VoIP service.

\section{IV.CONCLUSIONS}

In this paper, we presented a contention-free fully distributed coordination scheme proposed for IEEE 802.15.8 PAC. The focal point of design of the presented scheme is the scalability to avoid significant performance deterioration in a large number of proximate devices. The contention-free nature of the presented scheme is achieved by adopting orthogonal requests and responses. Simulation results for mean throughput per device with full buffer traffic model showed that mean throughput per device does not significantly deteriorate as the number of devices increases up to 1024 .

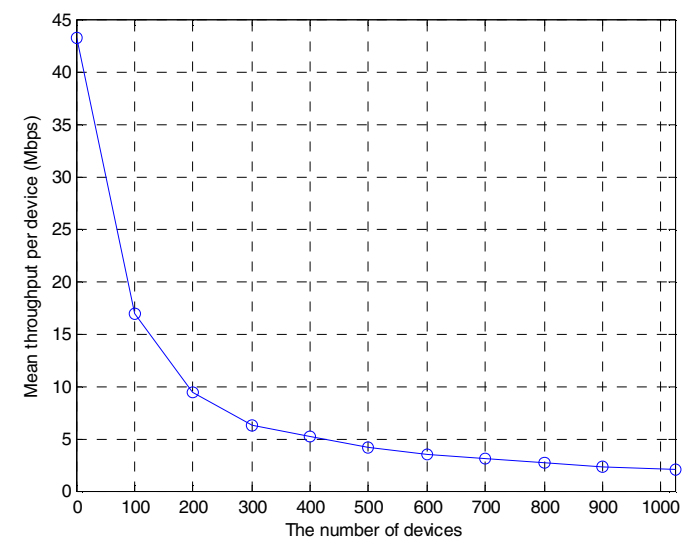

Figure 2. Mean throughput per device

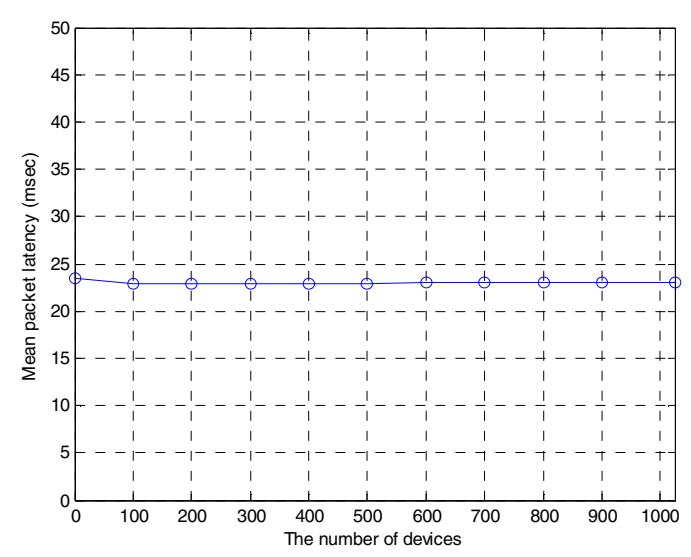

Figure 3. Mean latency of VoIP traffic

Moreover, the increase in mean packet latency with VoIP traffic is suppressed and remains almost constant for all the investigated number of devices. Therefore, we can conclude that the proposed scheme achieves scalability with the help of orthogonal requests and responses.

\section{ACKNOWLEDGMENT}

This research was funded by the MSIP(Ministry of Science, ICT \& Future Planning), Korea in the ICT R\&D Program 2013.

\section{REFERENCES}

[1] IEEE 802.15-12-0568-07-0008, TG8 Technical Guidance Document Available: https://mentor.ieee.org/802.15/documents. July. 2013.

[2] X. Wu, S. Tavildar, S. Shakkottai, T. Richardson, J. Li, R. Laroia, and A. Jovicic, "FlashLinQ: A Synchronous Distributed Scheduler for Peer-to-Peer Ad Hoc Networks", in Proc. 2010 Allerton Conf. Communication., Control, Computing, pp. 514-521, Sept. 2010.

[3] D. H. Kim, B. G. Choi, S. J. Bae, and M. Y. Bae, An adaptive connection scheduling method based on yielding relationship in FlashLinQ, ser. Lecture Notes in Computational Science and Its App.ICCSA 2013. Berlin, Germany: Springer, 2013, pp. 120-130.

[4] Seungkwon Cho, Hyungjin Kim, Seokki Kim, Soojung Jung, and Sungcheol Chang, "Text proposal of both MAC and PHY for PAC operating in synchronous mode (doc)", IEEE 802.15-13-0392-01-0008. Available: https://mentor.ieee.org/802.15/documents. July. 2013.

[5] IEEE 802.16m-08/004r5, Project IEEE 802.16m Evaluation Methodology. Available: http:// www.ieee802.org/16/tgm/docs/ 80216m-08_004r5.pdf. Jan. 2009. 